\title{
Ficha para el análisis tecno-tipológico de la industria ósea
}

\author{
Francisco Javier Muñoz Ibáñez *
}

El significado del término industria ósea tiene diversos conceptos que engloban a los objetos realizados sobre materias duras animales, basados en criterios tecnológicos, morfológicos y sociales. Así, se puede diferenciar la industria ósea poco elaborada, la industria ósea "clásica" y el arte mueble. Este último, sobrepasa los límites de este estudio.

En los últimos años hemos asistido a un notable incremento de los estudios sobre industria ósea, en donde han cobrado una mayor importancia aspectos tecnológicos, funcionales y experimentales; en relación con los procesos de obtención, transformación y utilización del utillaje y las potencialidades de la materia prima empleada. También, se ha producido una unificación de los criterios descriptivos, creándose una nomenclatura uniforme y abriéndose nuevas vías de investigación. En este proceso han tenido una gran importancia los coloquios organizados por H. Camps-Fabrer (1974, 1977 y 1979). Estos encuentros han conseguido reunir a los principales especialistas en este campo. Se ha logrado conferir al estudio de la industria ósea un carácter autónomo, siendo tratada de forma monográfica. De este modo, se ha convertido en un elemento de primera mano para el estudio del pasado, con un carácter similar al que tiene el análisis de la industria lítica para las sociedades cazadorasrecolectoras o el de la cerámica para las sociedades productoras prehistóricas. Este salto cualitativo en el estudio de la industria ósea ha hecho que se pase de una mera descripción a ser un elemento plenamente integrado en la investigación de un yacimiento o una zona concreta.

Este trabajo pretende ser una compilación de las diferentes metodologias aplicadas para el estudio de conjuntos industriales óseos, que per-

* Becario de F.P.I. Departamento de Prehistoria e Historia Antigua de la Universidad Nacional de Educación a Distancia. 
mita una recogida de datos clara y objetiva. Así, se ha elaborado una ficha de trabajo, siguiendo criterios fundamentalmente descriptivos, en donde se intenta identificar e individualizar las diferentes fases de los procesos de extracción del soporte y transformación del mismo, un análisis formal del útil y los diferentes atributos dejados por su uso. La reconstrucción de todas las etapas por las que ha pasado un objeto, desde la obtención de la materia prima hasta su abandono, puede ayudar a formular hipótesis relativas a la obtención y aprovechamiento de recursos, introducción de innovaciones técnicas, etc. Esta propuesta pretende colaborar a establecer las bases de un sistema de trabajo que vaya más allá de análisis estadísticos y tipológicos. Su puesta en marcha y los resultados obtenidos podrán ponderar su utilidad. Está especialmente ideada para materiales paleolíticos, pero la modificación de los campos necesarios posibilitará su ampliación a otros ámbitos cronoculturales. Actualmente, se está empleando para el estudio de la industria ósea de los niveles solutrenses del yacimiento de La Cueva de Ambrosio (Vélez Blanco, Almería). Su estructura permite ser aplicada a todo tipo de útiles óseos, con independencia de su grado de transformación, ofreciendo un sistema de análisis alternativo, de forma que no quede limitada al caso concreto del yacimiento para el cual fue formulada.

La ficha propuesta se articula en seis bloques que se analizan a continuación:

En el primer lugar se recogen los datos de control de cada objeto, que variarán en función de la metodología empleada en la excavación y la procedencia de la pieza. Además, se refleja:

- MATERIA PRIMA: sobre la que está realizada (hueso, asta, marfil, etc.).

- SOPORTE: soporte anatómico sobre el que se ha elaborado la pieza (candil, escápula, etc.). La identificación de este campo, como el siguiente, estará en función del grado de transformación y conservación del objeto.

- ESPECIE: animal al que pertenece dicho soporte.

Para completar este bloque, se procede al análisis tipométrico, basado en tres variables: longitud, anchura y espesor, expresados en milímetros, una vez orientado el objeto óseo. Tanto de la anchura como del espesor se realizarán dos mediciones; una en la zona mesial (Z.M.) y otra del valor máximo (+). Estas medidas son interesantes en si mismas para discriminar los módulos métricos más usados e intentar relacionar las posibles fracturas de uso con factores morfométricos. A partir de estas tres magnitudes se pueden definir una serie de índices muy útiles para comparar conjuntos de diferentes niveles o yacimientos y aproximarse a 
aspectos tecnológicos como capacidad de penetración de puntas de proyectil, durabilidad y resistencia de diferentes útiles y soportes, etc. Si bien los índices más utilizados son los de alargamiento y aplanamiento, se han recogido otros que pueden ser de utilidad:

\section{- INDICES DEFINIDOS POR H. DELPORTE:}

índice de Masividad: longitud máxima $\times$ espesor máximo.

Índice de Fragilidad: longitud total / espesor mesial. Índice de Alargamiento: longitud máxima / anchura máxima. Índice de Aplanamiento: anchura máxima / espesor máximo. Índice de Biselado: longitud del bisel /anchura máxima.

\section{- ÍNDICES DEFINIDOS POR H. CAMPS-FABRER:}

Índice de Ensanchamiento Total: anchura máxima $\times 100 /$ longitud total.

Índice de Espesor Total: espesor máximo $\times 100 /$ longitud total. Índice de Espesor Relativo: espesor mínimo $\times 100 /$ espesor máximo. Índice de Espesor Parcial: espesor medio mesial $\times 100 /$ longitud total. Índice de Aplanamiento: espesor medio mesial $\times 100 /$ anchura media mesial.

índice de Biselado: longitud del bisel / longitud total.

Según Camps-Fabrer (1976), para el cálculo de estos indices en las piezas fracturadas, habria que tomar las medidas originarias del objeto antes de su fractura. Sin embargo, en la mayoría de los casos no es posible reconstruir las dimensiones del útil, sobre todo en aquellos que presentan una fractura proximal/distal. "Se puede optar por realizar los cálculos sobre la longitud, anchura y espesor real del objeto, siempre y cuando se especifique claramente, para de este modo evitar interpretaciones erróneas" (Ruiz, MARTínez y ToRralba, 1983). Sin embargo, este sistema desvirtuaría en exceso los índices calculados. Por ello, sería más correcto calcular los índices en aquellos útiles que no presentan fractura o cuyas dimensiones puedan ser reconstruidas con un mínimo de fiabilidad para poder obtener conclusiones objetivas del análisis tipométrico y extrapolar estos datos a otros conjuntos.

En el siguiente bloque se analizan los PROCESOS DE EXTRACCIÓN; es decir, los procesos encaminados a la obtención del soporte para la fabricación de un útil. En función del grado de transformación de la superficie del objeto y su estado de conservación se podrá identificar o no la técnica o técnicas empleadas. En muchos casos resultará complejo llegar a discernir la técnica de extracción utilizada, ya que sus atributos morfológicos característicos pueden haber sido eliminados durante el proceso de elaboración o durante la utilización del objeto. La tradicional asocia- 
ción de determinadas tipologías con técnicas de extracción concretas, con independencia de sus atributos tecnomorfológicos; por ejemplo, varillas plano-convexas / aserrado, no siempre puede ser correcta. Para los distintos campos de este bloque se ha tomado como referencia el modelo establecido por Ruiz, Martínez y Torralba (1983), pero con una sistematización más simplificada, debido a que en muchas ocasiones es imposible concretar la técnica empleada. Así, aparecen las siguientes posibilidades:

- FRACTURA: bien sea por percusión, flexión o torsión. Es la más sencilla y simple de las técnicas de extracción. No obstante, presenta el inconveniente de ser la menos indicada para obtener soportes con una morfología predeterminada. Normalmente, constituye el primer paso en la obtención del soporte y suele ir acompañado de otros procesos. Los morfotipos característicos de esta técnica serían el astillamiento de la matriz y el soporte, las huellas de impacto dejadas por la acción del percutor y las fracturas por flexión o percusión dejadas en el soporte.

- ASERRADO: corte rectilíneo de una sola dirección y doble sentido realizado retiradamente con un instrumento afilado. Según Mújika (1990) las huellas del trabajo que deja esta técnica en el soporte son:

"- Huellas localizadas en la superficie cortical:

a) Las producidas por el raspado durante la preparación de la superficie a aserrar.

b) Las líneas de fuga, producidas al salirse el útil del interior del surco durante el aserramiento.

- Huellas localizadas en el plano de aserramiento:

a) Los planos de aserramiento, que conforman el surco, consisten en superficies planas que presentan líneas longitudinales: más o menos paralelas entre sí.

b) En la base del plano del aserramiento, frecuentemente, coincidiendo con el inicio del tejido esponjoso suele quedar como testigo un pequeño resalte que marca el límite entre la parte aserrada y la parte fracturada por flexión".

- ABRASIÓN: acción erosiva mediante la fricción de la materia prima contra un soporte abrasivo para adelgazarla. La huella más característica dejada por esta técnica, es la aparición de estrías en la superficie de abrasión, generalmente perpendiculares u oblicuas al eje longitudinal de la pieza; cuya profundidad y sección dependerá de la morfología del gano del abrasivo utilizado. Esta técnica extractiva puede complementarse al final del proceso con una fractura, generalmente por flexión, para la obtención del soporte. 
- FUEGO: «El punto seleccionado (...) para el corte era previamente expuesto al fuego, y una vez que estaba carbonizado esta parte era rascada con un pedazo de piedra aguzada. La muesca hecha (...) en forma de " $V$ " atravesaba la capa dura exterior penetrando en la parte blanda interna. Después de esto el hueso se rompia sin ningún esfuerzo" (SEMENOV 1981).

En el tercer bloque se describen los posibles PROCESOD DE TRABAJO que suponen una transformación antrópica del soporte. Tanto en este bloque como en los dos siguientes se ha puesto un especial énfasis en que la localización de cada campo sea lo más precisa posible. Asi, se ha dividido longitudinalmente el objeto óseo en ZONA DISTAL (Z.D.), ZONA MESIAL (Z.M.) y ZONA PROXIMAL (Z.P.). La diferenciación del fuste o zona mesial, de las zonas proximal y distal es sumamente difícil. La individualización de cada una de estas tres áreas puede hacerse adoptando una proporción determinada en relación a la longitud total del objeto. Cada una de estas tres zonas se subdivide a su vez en CARA SUPERIOR (C.S.), CARA INFERIOR (C.I.), BORDE DERECHO (B.D.) y BORDE IZQUIERDO (B.I.).

Se ha considerado las siguientes técnicas de acabado:

- PULIMENTO: acción abrasiva mediante el frotamiento del soporte contra una arenisca de grano fino para modificar su forma, eliminando las rugosidades de la superficie natural del soporte y restos de otros procesos de extracción o trabajo previos, para crear una superficie regularizada y lisa.

- ABRASIÓN: acción similar a la anterior, pero con el uso de una arenisca o piedra similar de grano grueso. En el soporte pueden llegar a observarse las líneas de fricción con el asperón y restos de trabajo previos.

- RASPADO: esta técnica consistente en extraer pequeñas astillas o virutas con un instrumento cortante. Los atributos característicos dejados por esta técnica serian finas estrias en la superficie de los objetos, generalmente longitudinales.

- PERFORACIÓN: orificio más o menos redondeado realizado mediante diferentes técnicas. Comprende tres etapas: preparación para controlar el punto, perforación y el acondicionamiento del orificio. En la descripción del objeto óseo habrá que especificiar si la perforación está realizada desde una o las dos caras, su sección y tipometría. Asimismo, se constatarán las huellas dejadas por los trabajos asociados a la perforación como la preparación previa de la superficie mediante un pulimento o raspado, estrías dejadas en el orificio por los útiles empleados, etc. 
- VACIADO: eliminación del tejido esponjoso del canal medular para la creación de cubiletes o enmangues. A efectos de rellenar los campos de localización de la ficha solo se contemplará ZONA DISTAL, MESIAL y PROXIMAL. Como en el caso anterior, en la descripción del objeto se analizará su sección y tipometría.

- RETOQUE: para este campo se ha seguido la tipología analítica de Laplace (1986). Según su orientación puede ser:

- Directo: afecta a la cara superior.

- Inverso: afecta a la cara inferior.

- Bifacial: afecta a ambas caras.

En cuanto al modo, pueden ser:

- Plano: cuando forma un ángulo inferior a $45^{\circ}$.

- Simple: cuando forma un ángulo cercano a $45^{\circ}$.

- Abrupto: cuando forma un ángulo superior a $45^{\circ}$ y cercano a $90^{\circ}$.

- Escamoso: retoque simple repetido sobre la misma zona, que le da una forma de escamas o escalonamientos.

La interpretación de los retoques es bastante problemática, ya que el comportamiento físico de la materia elástica es el mismo ante un retoque accidental que ante otro intencional (DAuvois 1974 y BoucHD 1974). Por ello, para que la finalidad prioritaria del retoque haya sido la adecuación del soporte para realizar un útil debe presentar una regularidad o alternancia no explicables por el azar o como resultado de otras actividades como la obtención del tuétano.

En el siguiente bloque, DESCRIPCIÓN MORFOLÓGICA, se analiza la superficie del objeto óseo y las morfologías generadas en los procesos de transformación del soporte. Se ha obviado recoger las formas y características de la superficie del objeto inherentes a los procesos anteriormente descritos. Es decir, es absurdo hablar aquí de una superficie pulimentada cuando anteriormente se ha constatado y localizado espacialmente el uso de la técnica del pulimento en la elaboración del útil. Así, aparecen los siguientes campos:

- TEJIDO ESPONJOSO: su localización, si lo hubiere, puede aportar datos significativos en relación con la parte activa de los instrumentos o las zonas que soportan una mayor tensión a modo de impacto o retención.

- CANAL MEDULAR: la importancia de este campo sería similar al del apartado anterior.

- SECCIÓN: se tomará la sección del objeto óseo tanto en la zona distal como en el fuste y la zona proximal. Este dato puede ser un interesante factor comparativo tanto entre diferentes niveles y yacimientos 
como elementos pertenecientes a un mismo tipo. Para rellenar este campo se puede optar por un código de números, un sistema de abreviaturas (opción elegida en este caso), etc. Entre las posibles secciones que pueden presentar los objetos óseos, se han recogido las siguientes:

$\begin{array}{llll}\text { CIRCULAR } & (\mathrm{C}) & \text { CÓNCAVO-CONVEXA } & \text { (CVCX) } \\ \text { SUBCIRCULAR } & \text { (SC) } & \text { CONVEXO-CÓNCAVA } & \text { (CXCV) } \\ \text { ELIPTICA } & \text { HELICOIDAL } & \text { (H) } \\ \text { LENTICULAR } & \text { (LE) } & \text { DISIMÉTRICA } & \text { (D) } \\ \text { OVOIDE } & (\mathrm{O}) & \text { TRIANGULAR } & \text { (T) } \\ \text { BILOBULADA } & (\mathrm{BL}) & \text { SUBTRIANGULAR } & \text { (ST) } \\ \text { TRILOBULADA } & (\mathrm{TL}) & \text { CUADRANGULAR } & \text { (CU) } \\ \text { TETRALOBULADA } & (\mathrm{TRL}) & \text { SUBCUADRANGULAR } & \text { (SCU) } \\ \text { BICONVEXA } & (\mathrm{BCX}) & \text { RECTANGULAR } & \text { (R) } \\ \text { BICÓNCAVA } & (\mathrm{BCV}) & \text { SUBRECTANGULAR } & \text { (SR) } \\ \text { PLANO-CONVEXA } & (\mathrm{PCX}) & \text { POLIGONAL } & \text { (P) } \\ \text { PLANO-CÓNCAVA } & (\mathrm{PCV}) & & \end{array}$

- LUSTRE: pátina o brillo que adquiere la pieza y que puede responder a varias causas: por uso, por motivaciones estéticas, como parte final del proceso de elaboración para que el objeto adquiera una mayor dureza (generalmente por exposición al fuego), como consecuencia de arrojarla al hogar al quedar inutilizada, etc. Por este motivo, se ha decidido darle un carácter meramente descriptivo al no poder incluirlo con toda seguridad dentro de los procesos de trabajo o como un atributo funcional.

- PUNTA: aguzamiento del extremo distal o proximal de un útil, en donde convergen los bordes y las caras de la pieza; eliminándose las aristas y creándose una superficie cónica y regularizada.

- APUNTADO: creación de una punta, pero sin llegar a conseguir una superficie cónica regularizada y bien diferenciada del fuste.

- BISEL: creación de uno o dos planos oblicuos que convergen con su opuesto, o llegan a cortarse en la prolongación imaginaria de los mismos.

- FILO: arista aguda creada por la convergencia de los dos bordes o las dos caras.

- MUESCA: concavidad o entalladura situada sobre el borde de un objeto.

- DIENTE: punta o resalto que aparece en los bordes de la pieza, generalmente perpendiculares $u$ oblicuos al eje longitudinal.

- ACANALADURA: hendidura longitudinal de sección más o menos cóncava. 
- GANCHO: morfología curva y más o menos puntiaguda en el extremo proximal del objeto para embutir a un astil.

- GRABADO: incisiones de trazo profundo y generalmente largo, en los que se denota una voluntariedad y no son consecuencia de procesos de extracción o elaboración.

- GRADO DE TRANSFORMACIÓN: después de un análisis morfológico de la pieza y teniendo en cuenta la información aportada por los dos bloques anteriores (PROCESOS DE EXTRACCIÓN Y DE TRABAJO) se procede a incluir en módulos arbitrarios: $(<35 \%)(35 \%-65 \%)(65 \%$ $99 \%)$ y $(100 \%)$; que porcentaje de la superficie del objeto óseo ha sufrido una transformación antrópica.

En el quinto bloque, DESCRIPCIÓN FUNCIONAL, se intentan recoger aquellos atributos morfologicos que se han generado en el utillaje óseo durante su utilización. Se parte, a priori, de un grupo reducido de caracteres formales que se podrán ampliar experimentalmente. Así, se han considerado los siguientes elementos:

ESQUIRLADO: "Superposición de levantamientos alargados y muy delgados, a menudo paralelos y escaleriformes" (Ruiz MARTínez y TORRALba 1983). Aparecen en el frente activo de útil y generalmente se relacionan con el elemento intermedio en la percusión indirecta o la talla por presión.

- MELLADO: presenta los mismos caracteres formales que el anterior, pero tiene un carácter mucho más marginal.

- EMBOTADO: redondeamientos de filos, puntas o aristas debido al uso del objeto. Normalmente, afecta a una zona localizada del útil, frente al rodamiento generalizado de la pieza como consecuencia de procesos mecánicos naturales o alteraciones físico-químicas.

- PIQUETEADO: huellas de impacto concentradas sobre la parte activa del objeto, que ocasionalmente pueden presentarse puntualmente por el resto de la superficie. Su presencia se asocia con la función de compresor-retocador.

- FRACTURA: rotura del objeto debido a su uso. Dentro de este campo, además de su localización espacial, se recogen las posibles causas por las que se ha podido producir, siempre que sea posible (FLEXIÓN, PERCUSIÓN, NO IDENTIFICADA). Asimismo, bajo la denominación de fractura moderna se agrupan aquellas fracturas que son recientes.

Se ha pretendido realizar una ficha técnica que permita tener una visión lo más completa posible de un objeto óseo y que permita la tabulación de los datos aportados de forma fácil y rápida, convirtiéndose en un elemento útil en el análisis objetivo entre diferentes niveles o yaci- 
mientos. Las posibles deficiencias que pueda presentar serán subsanadas mediante su aplicación a diferentes conjuntos industriales, hasta llegar a su estructuración óptima. El estudio de cada objeto se completaría con un dibujo del mismo en el dorso de la ficha. Aquí, sería interesante representar gráficamente las características morfotécnicas más importantes de cada pieza. Para ello, se ha elaborado un código de símbolos, algunos de los cuales se han aplicado a un ejemplo hipotético (fig. 2). No se han especificado aquellas convenciones gráficas tradicionalmente utilizadas en el dibujo arqueológico de útiles óseos, tal como el tejido esponjoso, canal medular, etc.

\section{BIBLIOGRAFIA}

Barandiaran, I. (1967): “El Paleomesolítico del Pirineo Occidental. Bases para una sistematización tipológica del instrumental óseo paleolítico", Monografías Arqueológicas 3. Zaragoza.

- (1981): Industria ósea. En: “El Paleolitico Superior de la Cueva del Rascaño (Santander)». (Centro de Investigaciones del Museo de Altamira. Ministerio de Cultura. Santander.

Cabrera, V. y Bernaldo De Quirós, F. (1978): “Principios de estudio de la industria de hueso poco elaborada", Trabajos de Prehistoria $n .^{\circ} 35$. Instituto de Prehistoria del C.S.I.C. Madrid.

CACHO, C. y RipolL, S.: “Industria ósea", en La Cueva de Ambrosio (Vélez-Blanco. Almeria) y su posición cronoestratigráfica en el Mediterráneo Occidenta/m. B.A.R. International Series n. ${ }^{\circ} 462$. Oxford.

BOUCHOD, J. (1974): Les traces de l'activité humaine sur les os fossiles. En Camps-Fabrer (organisateur).

CAmps-FabreR, H. (1974) (Organisateur): Premier colloque international sur l'industrie de l'os dans la prèhistoire. Université de Provence. De. de Université de Provence.

- (1977) (Organisateur): Méthodologie appliquée a l'industrie de lós prèhistorique. Colloque International du C.N.R.S. n. ${ }^{\circ}$ 580. Paris (Abbaye de Sébabque, 1976).

- (1979) (Organisateur): L'industrie en os et bois prèhistorique de cervidè durant le néolithique et l'age des metaux. C.N.R.S. París.

Dauvols, M. (1974): «Industrie osseuse prèhistorique et experimentations». En CampsFabrer (organisateur).

LAPLACE, G. (1972): "Recherches de typologie analytique», Origini II. Roma.

MuJikA, J. A. (1983): "Industria de hueso en la Prehistoria de Guipuzcoa", Munibe n. 35. San Sebastián.

- (1990): "La industria ósea durante el Paleolítico Superior: la técnica de aserramiento y extracción de lengüetas", Munibe $n .^{\circ} 42$. San Sebastián.

Newcomer, M. (1977): “Experimente in Upper Paleolthic bone work». En Camps-Fabrer (organisateur).

OTIE, M. (1974): "Caracteristiques inhérentes à l'analyse attributs de l'outillage osseux". En Camps-Fabrer (organisateur).

Ruiz, E.; Martinez, C. y Torralba, F. (1983): “Ensayo metodológico para el estudio de materiales óseos", Antropología y Paleoecología Humana $n .^{\circ} 3$. Granada.

SeMENov, S. A. (1981): Tecnologia prehistórica. Ed. Akal, Madrid.

VorUz, J. L. (1979): “Typologie analytique d'industries osseuses neolitiques". En CampsFabrer (organisateur). 


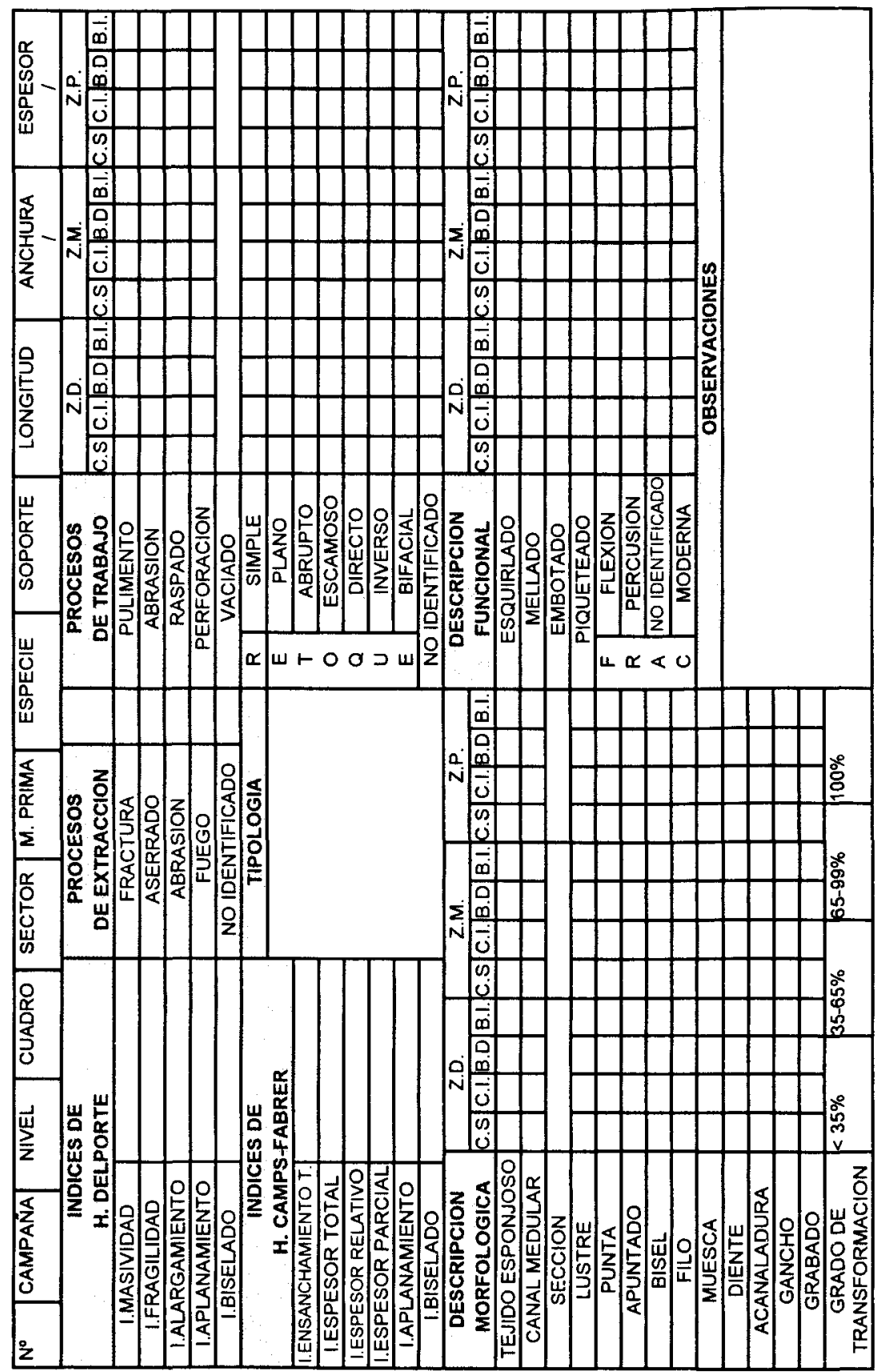

Fig. 1. Ficha para el análisis tecnotipológico de la industria ósea. 

C. $S$.
B. I.
C.I.
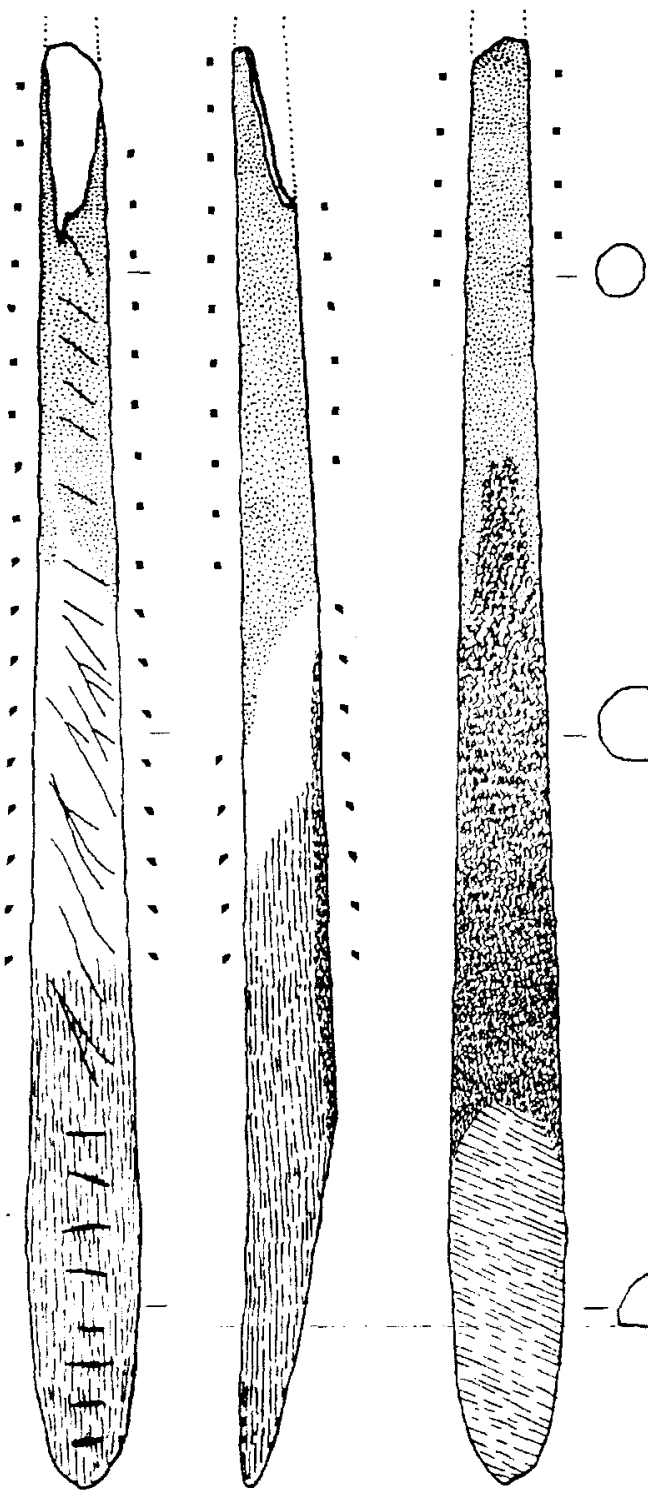

\section{LEYENDA}
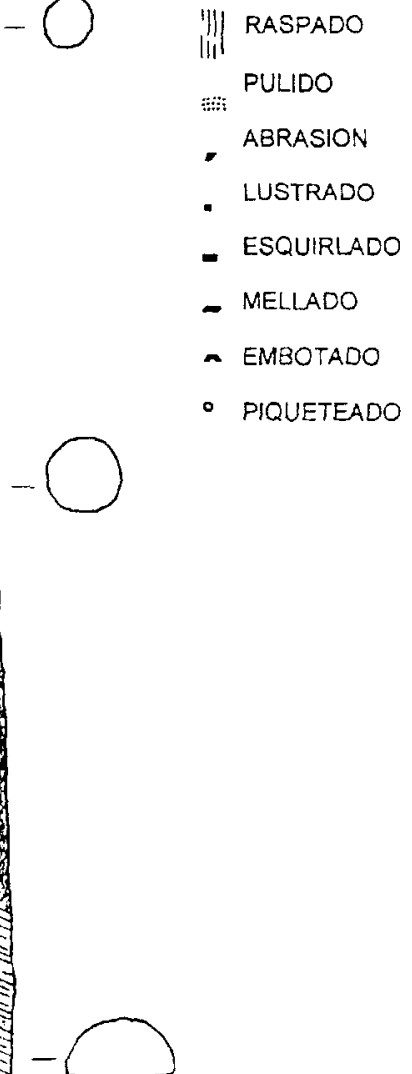

Fig. 2. Código de simbolos de los campos descritos en la ficha, complemento del dibujo arqueológico, y ejemplo de su utilización. 